\title{
HURRICANE INTENSITY PREDICTABILITY
}

\author{
by Chanh Q. Kieu and Zachary Moon
}

\section{A chaotic attractor at the hurricane maximum intensity stage is captured in an axisymmetric hurricane model, thus suggesting an upper limit on the accuracy of hurricane intensity forecasts at the 4-5-day lead times.}

A mong various weather systems in the atmosphere, hurricanes possess distinct behaviors due to their approximate axisymmetric structure ${ }^{1}$ and thermodynamic feedbacks at the mesoscale, which render their characteristics unique as compared to other atmospheric systems, such as tornadoes, convective cells, and frontal systems. An important result based on this axisymmetry is the well-known steady-state solution for the maximum potential intensity $\left(\mathrm{MPI}^{2}\right)$ that a hurricane can attain under a prescribed thermodynamic condition, which is given by (Emanuel 1986, 2003)

$$
V_{m}^{2}=\frac{C_{D}}{C_{E}} \frac{\left(T_{s}-T_{o}\right)}{T_{s}}\left(k_{s}^{*}-k_{a}\right)
$$

where $T_{s}$ is the sea surface temperature (SST); $T_{o}$ is the mean outflow temperature near the tropopause; $C_{D, E}$ are the surface drag and enthalpy exchange

\footnotetext{
${ }^{1}$ The axisymmetry of hurricanes in this study is understood in the sense of the zero-order linearization, in which all asymmetric forcings can be parameterized in terms of azimuthally averaged variables (Willoughby 1979).

${ }^{2}$ In this study, the phrase "maximum potential intensity" refers to the maximum axisymmetric tangential wind that a storm can attain at its statistical quasi-equilibrium stage, not the instantaneous intensity during the storm transient adjustment or statistical fluctuations of intensity.
}

coefficients, respectively; and $k_{s}^{*}$ and $k_{a}$ are the moist entropy at the ocean surface and the adjacent atmospheric surface layer, respectively. While the MPI solution [Eq. (1)] has proved to be helpful in a broader context of the hurricane-climate connection under different climate change scenarios or intensity outlooks (e.g., Henderson-Sellers et al. 1998; Knutson et al. 2010; Walsh et al. 2015), it has not been demonstrated whether this steady-state solution is stable. In fact, it would be rather catastrophic if the MPI solution were found to be unstable or if there exist multiple stable points, because this would then imply that the MPI solution cannot be reached and therefore has little practical value. Numerous idealized simulations with either full-physics models or idealized simulations with axisymmetric hurricane models have shown that model-simulated vortices do not settle down to the MPI solution but rather display strong intensity oscillations around the theoretical equilibrium given by Eq. (1) (e.g., Rotunno and Emanuel 1987; Bryan and Rotunno 2009; Hakim 2011, 2013; Brown and Hakim 2013). This poses an important question of whether there exists any limit cycle or an unstable regime that prevents hurricanes from approaching their MPI equilibrium. Of course, the strong variation around the MPI equilibrium could be inherently related to the fact the theoretical MPI solution is valid only under idealized assumptions 
such as the gradient wind balance, rotational axisymmetry, or simplified radial momentum budget in the planetary boundary layer. However, the fact that the MPI solution as given by Eq. (1) could provide a reasonable statistical upper bound of intensity for the majority of observed storms (e.g., Emanuel 2000; Bryan and Rotunno 2009) suggests that the MPI limit does contain important implications about the hurricane intensity equilibrium that the steady-state theory could not reveal.

STABILITY OF MPI EQUILIBRIUM. Stability of the MPI equilibrium was recently examined in a framework of the hurricane-scale dynamics, in which the asymptotical stability of the MPI equilibrium under the wind-induced surface heat exchange (WISHE) mechanism was demonstrated (Kieu 2015). A low-order hurricane-scale model [referred to as the hurricane-scale dynamical (HSD) model] proposed by Kieu (2015) is based on dynamics of several hurricane basic scales, including the scale of the maximum surface wind $V_{m}$, the scale of the maximum vertical motion within the inner-core region $W_{m}$, and the scale of the warm anomaly in the eye center that is represented by buoyancy variable $B_{m}$. The essence of this HSD model is that these scales are not independent but strongly governed by the hurricane dynamics and thermodynamics, which can be shown to evolve in time according to the following set of nondimensionalized constraints:

$$
\begin{aligned}
\dot{v} & =v w-v^{2}, \\
\dot{w} & =-s v^{2}+s b, \\
\dot{b} & =-b w+v^{2}-\kappa b,
\end{aligned}
$$

where $v, w$, and $b$ denote nondimensional values of $V_{m}$, $W_{m}$, and $B_{m}$, respectively; $s$ is a parameter representing the squared ratio of the depth of the troposphere to

AfFiliations: Kieu AND Moon-Atmospheric Science Program, Department of Geological Sciences, Indiana University, Bloomington, Indiana

CORRESPONDING AUTHOR: Chanh Kieu, Department of Geological Sciences, Indiana University, I00I East 10th Street, GY428A Geological Building, Bloomington, IN 47405-I405 E-mail: ckieu@indiana.edu

The abstract for this article can be found in this issue, following the table of contents.

DOI:10.II75/BAMS-D-15-00168.1

A supplement to this article is available online (10.1175/BAMS-D-15-00168.2)

In final form 27 February 2016

(02016 American Meteorological Society the depth of the planetary boundary layer; and $\kappa$ is a constant representing the Newtonian cooling. An interesting property of this HSD system is the existence of a unique stable critical point given by $v \approx(1-\kappa / 2)$, $w \approx(1-\kappa / 2)$, and $b \approx(1-\kappa / 2)$ for $\kappa \sim O\left(10^{-1}\right)$, which corresponds indeed to the MPI equilibrium [in the absence of radiative cooling, the point $v=1$ corresponds exactly to $V_{m}$ given by Eq. (1); see Kieu 2015]. Examination of the local stability of this critical point shows that although the radiative forcing could modify the basin of the attraction toward the MPI equilibrium, the equilibrium is structurally stable and is a hyperbolic sink; all neighbors of the MPI will converge spirally to the MPI equilibrium regardless of the vortex's initial conditions (Fig. 1).

Despite the simplicity of the HSD model, two significant implications follow from the resulting MPI stability. First, the MPI equilibrium should no longer be characterized just in terms of $V_{m}$ (i.e., $v=1$ ) as often presumed in previous studies, but it should take into account conditions for both the warm-core anomaly $(b=1)$ and the vertical motion in the central storm region $(w=1)$ as well. This strong constraint among the three scales $(v, w, b)$ explains why a slight perturbation in the warm-core anomaly or fluctuations in the secondary circulation can easily cause a hurricane's intensity to oscillate strongly around the equilibrium, even when $V_{m}$ matches exactly the MPI value. Such a constraint in the hurricane scales may also justify why the theoretical MPI has never been captured in previous numerical simulations, which inherently contain many sources of vertical motion perturbations and temperature anomalies.

Second, the unique sink of the HSD system implies that the hurricane end fate is nothing but a stable point, which is determined by the large-scale environment regardless of the initial conditions. As a demonstration of the stability and the uniqueness of the MPI equilibrium, Fig. 1 shows the flow orbits obtained from numerical integrations of the HSD system [Eqs. (2a)-(2c)] with several different initial conditions; all are indeed spirally attracted toward the MPI equilibrium located at the point $(1,1,1)$ in the phase space of $(v, w, b)$. Of the four example orbits, note in particular the strong initial wobbling of the red orbit for which the initial value of $V_{m}$ is set equal to the exact MPI value [i,e. $v(0)=1$ ], but the initial warm anomaly $B_{m}$ is half of the critical value [i.e., $b(0)=0.5$. This specific case resembles a situation in which one initializes a hurricane model with an initial vortex whose $V_{m}$ matches the observed intensity, but the initial warm anomaly at the core of the vortex is not consistent with the wind structure. In 
this scenario, it is expected that the vortex would experience a rapid adjustment before approaching the maximum intensity limit similar to that seen in Fig. 1, thus resulting in large initial intensity errors. Despite this initial adjustment, the final stage of the hurricane development is still the same MPI equilibrium as dictated by the HSD system as long as the large-scale conditions are conducive to the hurricane development.

Of interest is that the existence of the unique MPI sink could shed light on a standing issue in current operational hurricane models, which all exhibit rapid growth of intensity errors during the first $36-48 \mathrm{~h}$, but the intensity errors saturate quickly after 3 days regardless of numerical models or initial conditions. Figure 2 shows an example of verification of real-time intensity forecasts in the northwestern Pacific (WPAC) basin for five different operational models during 2012-14. Here, the verification for the WPAC basin is presented because of the larger sample size of strong storms that will facilitate our subsequent analysis of the MPI limit and chaotic attractor. Except for the Naval Global Environmental Model (NVGM), which possesses an initial intensity error as large as the 4-5-day errors, all other models display the most rapid intensity error growth during the first $48 \mathrm{~h}$ despite very different vortex initial conditions and numerical modeling systems. In light of the HSD dynamics, such behavior is a consequence of the fact that the hurricane development does not have any other choices. As soon as favorable conditions for
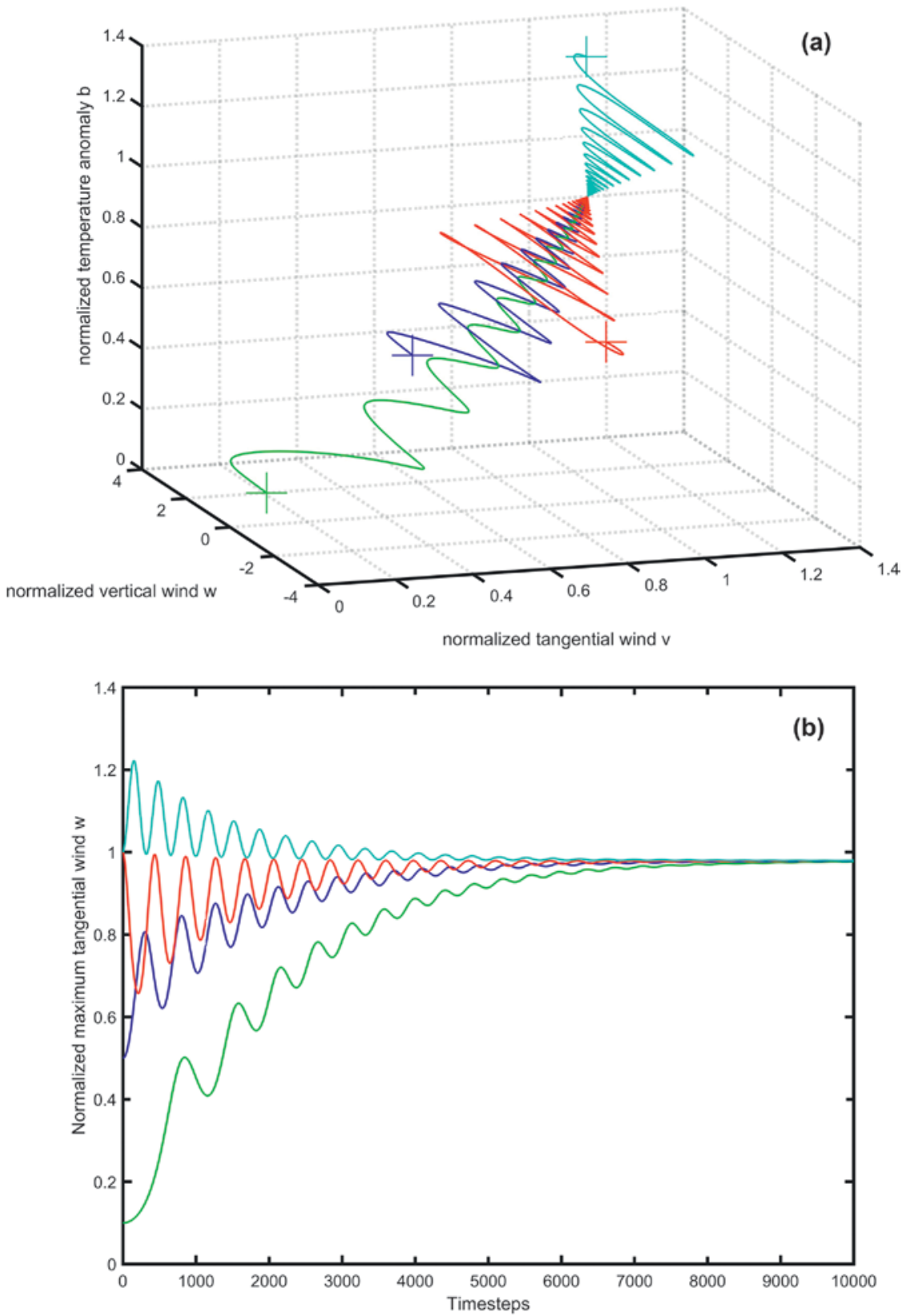

FIG. I. (a) Orbits of flows in the phase space of the nondimensionalized $V_{m}, W_{m}$, and $B_{m}$ for four different initial conditions: $(0.1,0.1,0.1)$ (green), $(0.5,0.5,0.5)$ (blue), (I.0, 0.5, 0.5) (red), and (I.0, I.4, I.4) (cyan). The orbits are obtained from numerically integrating the HSD system [Eqs. (2a)-(2c)] with parameter $s=100$, which corresponds to a hypothetical troposphere with a tropopause height of $18 \mathrm{~km}$, a planetary boundary layer height of $2 \mathrm{~km}$, and a surface drag coefficient $C_{d}=10^{-3}$. (b) Projections of the orbits in (a) along the direction of $V_{m}$.

the hurricane feedback are ensured, an initial vortex will be forced to approach an MPI limit at which its absolute angular momentum can be balanced by the frictional forcing. Because of this convergence of hurricane intensity toward the same equilibrium even along highly different orbits, the rate of the intensity 


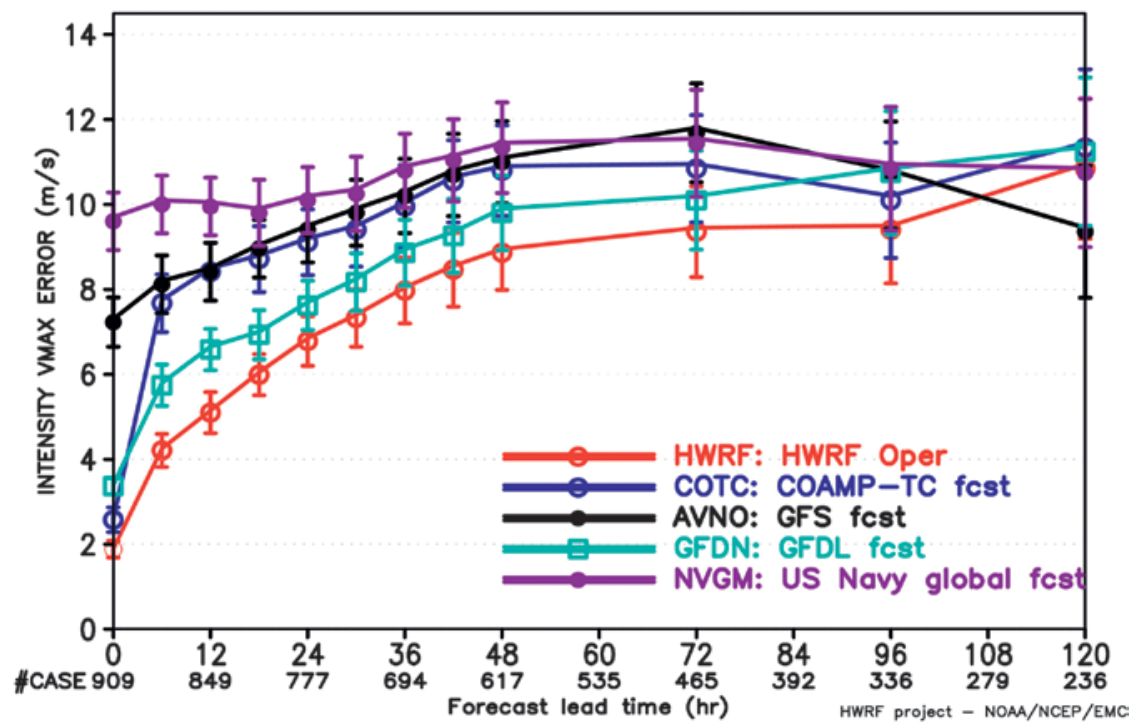

FIG. 2. Verification of the real-time hurricane intensity forecasts $\left(\mathrm{m} \mathrm{s}^{-1}\right)$ in the WPAC basin during 20I2-I4 for the HWRF model (red), the U.S. Global Forecast System model (GFS; black), NVGM (purple), the Princeton GFDL model (cyan), and the COAMPS-TC model (blue). Numbers below each forecast lead time denote the number of cases verified. Error bars denote $\mathbf{9 5 \%}$ confidence intervals.

error growth will be gradually flattened out at longer ranges as seen in Fig. 2.

It should be noted that for real storm development, it is almost certain that few, if any, storms could eventually reach their MPI due to continuous changes in the ambient environment. However, as long as the hurricane's intensity approaches the same intensity limit, both the forecasted and observed intensities will ultimately converge, and the intensity errors will therefore diminish at longer ranges. Such convergence toward the MPI equilibrium justifies the fact that various idealized experiments in previous studies with considerably different vortex initial strengths, structures, and approximations all share a similar hurricane structure at the mature stage (see, e.g., DeMaria and Schubert 1985; Rotunno and Emanuel 1987; Wang 2001; Hendricks et al. 2004; Yang et al. 2007; Bryan and Rotunno 2009). From this perspective, the existence of the unique MPI stable point is noteworthy, as it implies that a hurricane's intensity and structure depend more on the large-scale environment at the longer ranges than on the hurricane's initial conditions.

CHAOTIC MPI ATTRACTOR. While the stable and unique MPI equilibria may justify the slower rate of error growth at the long lead times as discussed above, the intensity errors at 4-5-day lead times do not approach zero as would be expected if the MPI equilibria were truly unique. Instead, the 4-5-day intensity errors are consistently around $8-10 \mathrm{~m} \mathrm{~s}^{-1}$ as seen in Fig. 2, for which the HSD system cannot explain. From a practical standpoint, such intensity errors are apparently unavoidable even in the framework of the hurricane-scale dynamics because of several factors, such as the constant movement of hurricanes under largescale environmental steering flows, dry-air intrusion, strong vertical wind shear, and landfall, which cuts off the energy input. Therefore, the MPI has to continuously adjust to the new environment such that hurricanes may have little chance to settle down to their equilibrium. On the other hand, the long-range errors may be related to some hidden chaotic nature of the MPI equilibrium in higher dimensions that the low-order hurricane-scale framework cannot account for due to the lack of detailed processes in the HSD model, such as cloud radiative feedback, microphysics, and small-scale dynamical processes.

To address the relative impacts of the large-scale fluctuations on the MPI stability versus potential existence of a chaotic attractor in a more general setting, a full-physics axisymmetric model developed by Rotunno and Emanuel (1987, hereinafter the RE model) is used for a set of 1,000-day simulations (see supplement for the description of the RE model: http://dx.doi .org/I0.II75/BAMS-D-I5-00168.2). For such a fullphysics model with $\sim 5 \times 10^{4}$ degrees of freedom, ${ }^{3}$ it is no longer feasible to expect a single equilibrium point for orbits to settle down. Instead, the most optimistic scenario for such a high-dimension model is that the model would display an attracting set that the hurricane orbits will approach after some period of time instead of a pointlike attractor. The reason behind such expectation of an attracting set at the MPI equilibrium in full-physics models is because

\footnotetext{
${ }^{3}$ The number of degrees of freedom for this model is derived from the default configuration of the RE model, which consists of $200 \times 45$ grid points in the $(r, z)$ directions, and five prognostic variables.
} 
numerical models often implement numerous upper/ lower bounds for different physical parameterizations, such as the radiative forcing caps, truncated tendencies and forcings, or the maximum lapse rate, to ensure the model stability. In this sense, numerical models are genuinely a bounded dynamical system. In addition, various averaging or filters that act as absorption of unrelated waves could render the numerical models dissipative after sufficiently long integration. Viewing from this perspective, the existence and closure for the bounded attracting sets are fully ensured (Ott 2002), and it is thus natural to expect that the full-physics model should display a finite-volume attracting set rather than a pointlike attractor as dictated by the HSD system. Whether this type of an attracting set could possess chaotic features is an issue that depends further on the denseness property and the existence of positive Lyapunov exponents, which may be specific to each individual model, physical parameterization, boundary treatments, or model vertical and horizontal resolution.

Because it is impossible to represent flows in the phase space of dimension $\sim 5 \times 10^{4}$, Fig. 3 shows the flow orbits in a reduced phase space consisting of the three fundamental scales $V_{m}, W_{m}$, and the maximum temperature anomaly at the vortex center $T_{m}$. Here, different colors in Fig. 3 represent a set of 1,000-day simulations with different vortex initial intensities, which ranges from $V_{m}=12$ to $50 \mathrm{~m} \mathrm{~s}^{-1}$. Such representation of the flow orbits in the reduced space is meaningful because the denseness of any chaotic attractor ensures that its projection onto a subspace is also chaotic (Lorenz 1963; Ott 2002). While there is no single pointlike attractor as anticipated, it is seen in Fig. 3 that all orbits are quickly pulled toward a specific region in the phase space of $\left(V_{m}, W_{m}, T_{m}\right)$ due to the bounded property of the RE model, with its center located roughly at $V_{m} \sim 66 \mathrm{~m} \mathrm{~s}^{-1}, W_{m} \sim 5 \mathrm{~m} \mathrm{~s}^{-1}$, and $T_{m} \sim 20 \mathrm{~K}$ (referred to as the MPI attractor ${ }^{4}$ ). One notices also the strong wandering of the vortex intensity during the first several days into integration with $V_{m}$ reaching as high as $100 \mathrm{~m} \mathrm{~s}^{-1}$ before settling down to the MPI attractor, which is somewhat similar to the intensity variation in the low-order model (cf. Fig. 1). The large fluctuation in the vortex intensity during the transient period, often linked to the supergradient intensity, is seen even for the case with initial $V_{m}=50 \mathrm{~m} \mathrm{~s}^{-1}$ (green dots), which is fairly

\footnotetext{
${ }^{4}$ Technically, an attractor contains more structure than the attracting set. In this study, we will refer to the attracting set displayed in Fig. 2 later as an MPI attractor, based on an assumed denseness of the flow orbits in the RE model.
}

close to the MPI limit of $\sim 66 \mathrm{~m} \mathrm{~s}^{-1}$. Such an initial adjustment is likely because the RE model vortex is initialized with a simple vortex structure that has the tangential wind maximum at the surface and linearly decreased upward, and the temperature anomaly derived from the thermal wind relationship, whereas the initial secondary circulation is not taken into account (Rotunno and Emanuel 1987). In light of the low-order HSD model, this vortex initialization produces an initial inconsistency among $V_{m}, W_{m}$, and $B_{m}$, and the vortex intensity thus varies strongly before approaching the quasi-equilibrium stage.

Unlike the Lorenz attractor that swiftly exhibits beautiful butterfly wings after a few hundred iterations (Palmer 1993), one further notices in Fig. 3 that there is no limit cycle or any specific fractal structure for the MPI attractor in the RE model, even after a long integration of 1,000 days. Instead, flows in the RE model are trapped within a volume depicted by the cloud of points in the phase space of $\left(V_{m}, W_{m}\right.$, $T_{m}$ ) with a size of $\sim 8 \mathrm{~m} \mathrm{~s}^{-1}$ as projected onto the $V_{m}$ dimension regardless of the initial vortex conditions (here, the size of the attractor is defined as a standard deviation with respect to the 1,000-day mean value; cf. Figs. 3 and 4 a).

The convergence of different initial conditions toward the same MPI attractor in the RE model is consistent with the conclusions drawn from the HSD model (cf. Fig. 1) and contains significant implications for the hurricane predictability limit. Recall from Lorenz's definition of the range of predictability as the time interval within which forecast errors would not exceed the difference between randomly chosen states. As shown in Fig. 3, such a difference according to Lorenz's definition is $\sim 8 \mathrm{~m} \mathrm{~s}^{-1}$ for experiments with very different initial vortex conditions, and so the limit of $8 \mathrm{~m} \mathrm{~s}^{-1}$ in this sense could indeed represent the intensity error saturation in the RE model as defined by Lorenz (1969). Of course, the four experiments shown in Fig. 3 may not fully characterize the entire set of randomly selected initial states, but they could well cover the variation of the vortex initial intensity for practical purposes. In fact, our sensitivity experiments with many different combinations of initial conditions in the RE model do exhibit the same MPI attractor (not shown), thus suggesting the limit of $8 \mathrm{~m} \mathrm{~s}^{-1}$ as an error saturation threshold in the RE model, below which the intensity error cannot be reduced further by improving the model's initial conditions.

Of significance is that the overall boundedness and denseness of the volume surrounding the MPI equilibrium gives us a hint that this MPI attractor may possess sensitive dependence on initial 


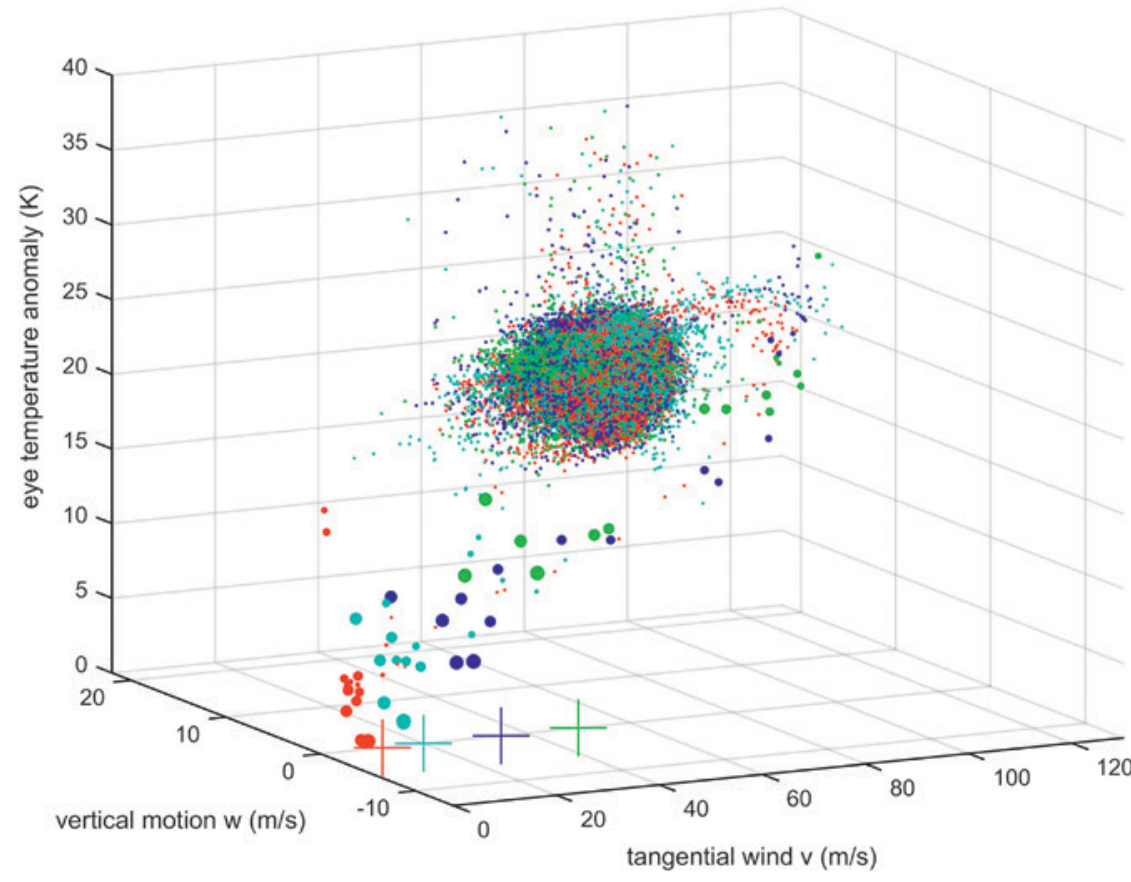

FIG. 3. Orbits of the hurricane states in the phase space of $\left(V_{m}, W_{m}, T_{m}\right)$ obtained from the I,000-day simulations, using the RE axisymmetric model with four different initial conditions: $V_{m}=12$ (red), 20 (cyan), 30 (blue), and $50 \mathrm{~m} \mathrm{~s}^{-1}$ (green). Crosses denote the vortex initial intensity, and the large dots denote the vortex states during the first $72 \mathrm{~h}$ into integration.

conditions, which could inhibit us from reducing the hurricane intensity errors after hurricanes enter their MPI regime.

To shed light onto the initial condition sensitivity inside the MPI attractor in the RE model, the leading Lyapunov exponent is numerically estimated for 500 cycles starting after 5 days into integration, when the simulated vortex roughly reaches its statistical equilibrium (Fig. 3a). Here, the leading Lyapunov exponent is defined as

$$
\lambda_{m}=\lim _{t_{m} \rightarrow T_{\infty}} \lim _{\epsilon \rightarrow \epsilon_{\infty}} \frac{\left\|\mathbf{v}^{\epsilon}\left(t_{m}+\tau\right)-\mathbf{v}\left(t_{m}+\tau\right)\right\|}{\|\epsilon\|},
$$

where $\mathbf{v}=(u, v, w, T, q)$ is the model state vector; $\boldsymbol{\epsilon}$ is a perturbation vector; $\mathbf{v}^{\epsilon}$ is the perturbed state; $\|. .$.$\| is$ the energy norm, which is defined as

$$
\|\mathbf{v}\|=\sum_{i} \sqrt{\left(u_{i}^{2}+v_{i}^{2}+w_{i}^{2}+C_{p} T_{i}+L_{v} q_{i}\right)}
$$

with the index $i$ running over all the model's grid points; $T_{\infty}=500$ days; $\boldsymbol{\epsilon}_{\infty}$ is a prescribed numerical limit of the perturbation scale at each iteration; $\tau=3 \mathrm{~h}$ is a fixed time interval for cycling the perturbation; and the subscript $m$ indicates the $m$ th number of iterations. Technically, $\boldsymbol{\epsilon}_{\infty}$ needs to approach zero as $T_{\infty} \rightarrow \infty$. Because of the numerical limitation with
$T_{\infty}=500$ days, $\boldsymbol{\epsilon}_{\infty}$ is set to $10^{-3} \mathrm{~m} \mathrm{~s}^{-1}$ in all of the experiments in this study. Note also that the vector norm in Eq. (3) is computed for all prognostic variables instead of limiting within the threedimensional phase space $(v, w, b)$ as shown in Fig. 1 . This is to ensure that the numerical leading exponent $\lambda_{m}$ could reflect the true growth rate of the intensity perturbation in the RE model.

It is seen in Fig. 4 that the MPI attractor indeed possesses a positive leading Lyapunov exponent with an averaged value of $\sim 3$. Such a positive Lyapunov exponent and the assumed boundedness and denseness strongly indicate the chaotic nature of the MPI attractor in the RE model instead of a simple attracting set; a small perturbation imposed on the flow trajectory inside the MPI attractor will amplify with a factor of $\sim e^{3}$ and soon spread out over the entire attractor space after approximately $3 \mathrm{~h}$ into integration. A direct consequence of this finding is that any fluctuation in the ambient environment after a storm reaches its mature stage could quickly produce as large of a variation in the storm intensity as the entire attractor space after just a few hours. From the standpoint of numerical model forecasts, this means a small perturbation introduced to hurricanes at their mature stage will lead to strong fluctuation in a hurricane's intensity, and there is no effective way to prevent this intensity fluctuation. As a result, there appears to be little hope of controlling the hurricane intensity error once they enter their MPI attractor, at least from the perspective of the RE model.

The chaotic property of the MPI attractor demonstrated in Fig. 4 leads to an intriguing dilemma. On one hand, the existence of the MPI attractor implies that any small change during the early stages of the storm development will not generally have much of an impact on the later hurricane development; all vortex initial conditions will be pulled toward the MPI attractor after some period of time. Such an MPI attractor has some real implication to the development of numerical models, as this demonstrates that 

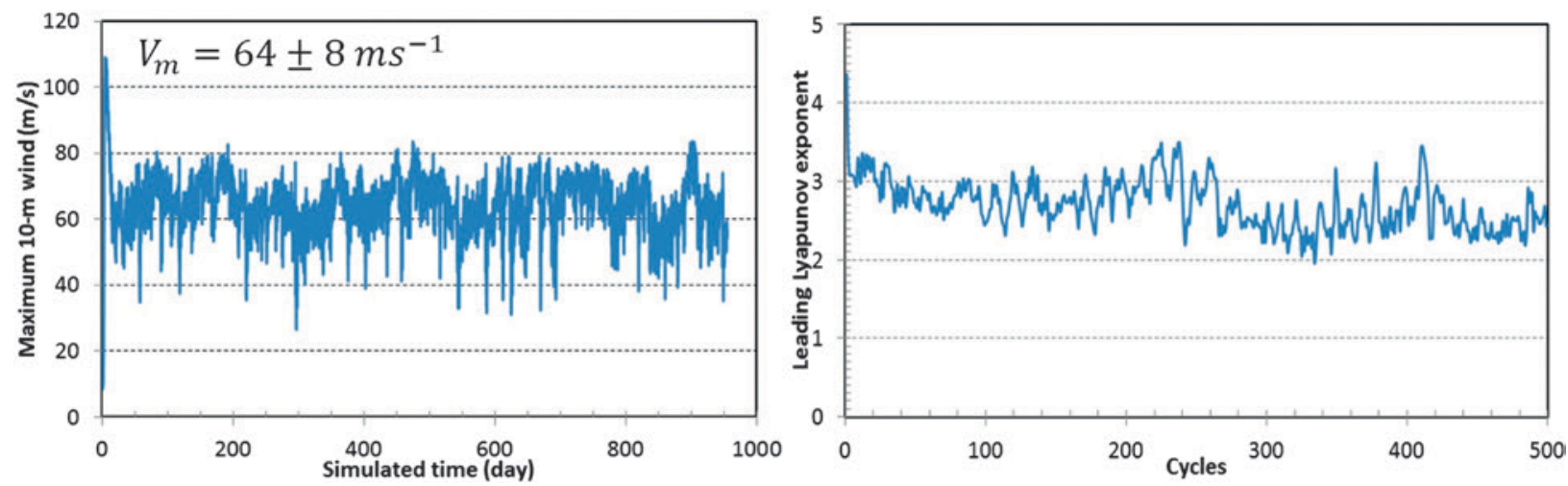

FIG. 4. (left) Time series of the maximum surface wind $\left(\mathrm{m} \mathrm{s}^{-1}\right)$ in a 1,000 -day simulation using the RE axisymmetric model. (right) The leading Lyapunov exponent during an iteration of 300 cycles starting 5 days into integration after the model vortex enters its MPI attractor.

a hurricane's intensity in a numerical model will not increase indefinitely but tend to approach a limit regardless of how we initialize models, implement new physical parameterization schemes, higher model resolution, or different numerical algorithms. Basically, the model states will be trapped inside an attractor whose characteristics are determined by the large-scale environment instead of storms' initial conditions.

On the other hand, the chaotic nature of the MPI attractor suggests that any small fluctuation in the large-scale environment will rapidly drift the hurricane's intensity away, with intensity variations as large as the entire MPI attractor space. There is no simple way to control such intensity fluctuation. For example, a small perturbation of a wind component of $0.5 \mathrm{~m} \mathrm{~s}^{-1}$ will amplify to as large as $9 \mathrm{~m} \mathrm{~s}^{-1}$ in just $3 \mathrm{~h}$ according to the RE model. This may justify the constant range of the $8-10 \mathrm{~m} \mathrm{~s}^{-1}$ intensity errors at the 5-day lead time during the last 30 years as seen in Fig. 2, despite the significant reduction in the track forecast errors. In this sense, the chaotic property of the MPI attractor imposes a true upper bound on our future effort of improving the accuracy of hurricane intensity forecasts.

REAL-TIME REALIZATION. Although the RE model could provide an estimate for the intensity error saturation of $\sim 8 \mathrm{~m} \mathrm{~s}^{-1}$ at the mature stage, how this error saturation is further realized and interpreted for real-time intensity predictability requires further examination. Understanding the implication of such error saturation to the range of the predictability limit in real-time intensity forecasts will directly address an important question of how far into the future a dynamical model can predict the hurricane's intensity. While Lorenz (1969) suggested a 2-week range for an initial error to approach an error saturation limit in the two-dimensional barotropic flow framework, this 2-week limit turns out to depend critically on the underlying assumption of the basic energy spectrum. Recent studies by Rotunno and Snyder (2008), Durran and Gingrich (2014), and Judt et al. (2016) indicated that a simple change from the $-5 / 3$ spectrum to the -3 spectrum of the background energy could result in a radical shift from limited to unlimited predictability. With the unique rotational dynamics of hurricanes, it is thus natural to expect a much different range of predictability for hurricane intensity forecasts from the general 2-week limit obtained in Lorenz's (1969) model.

Before interpreting the chaotic MPI attractor obtained from the RE model for real-time intensity errors, it should be recalled that real-time forecasts by operational models contain statistics of all cycles with different storm initial intensities at all stages of development instead of monotonic growth from the beginning to the maximum intensity limit as in idealized experiments. In addition, real hurricanes constantly move into colder SSTs and stronger vertical wind shear at higher latitudes or simply make landfall, and so often weaken rapidly before even entering the MPI attractor if they move too fast or are too close to coastlines. In this regard, the real-time 4-5-day intensity errors do not always imply the errors inside the MPI attractor. To reduce the negative impact of the mixed-cycle statistics on the predictability limit in real-time verification, Fig. 5 shows the verification of a hurricane's intensity similar to that shown in Fig. 2, but the verifications are carried out only for cycles with the observed initial intensity of category 2 and above (i.e., $V_{m} \geq 47 \mathrm{~m} \mathrm{~s}^{-1}$, or $\sim 95 \mathrm{kt}$ ). Such an initially strong intensity stratification is to ensure that hurricanes approximately enter their MPI 


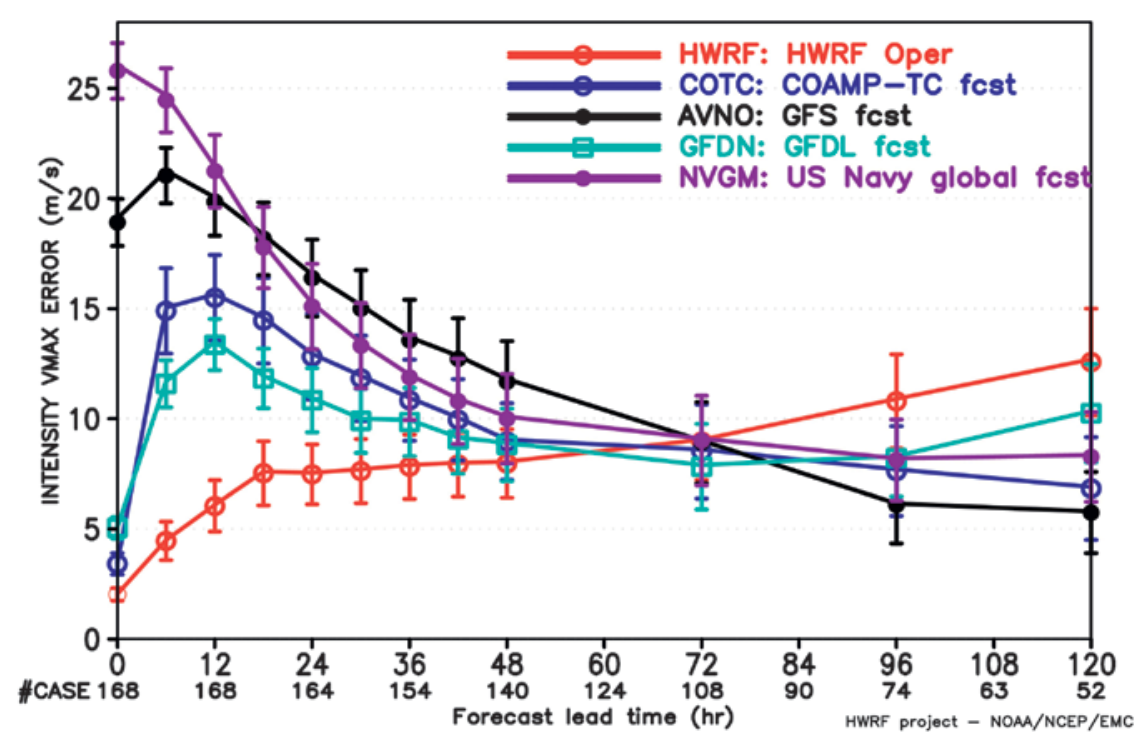

FIG. 5. As in Fig. 2, but for verification of hurricanes with an initial intensity greater than $47 \mathrm{~m} \mathrm{~s}^{-1}$.

attractor region. ${ }^{5}$ Note that this selection of initially strong storms in the real-time verification does not entirely eliminate cases in which the real hurricanes weaken too quickly as they move into colder SST or make a landfall. Nevertheless, it could at least highlight the growth of intensity errors of strong storms that we can at most infer inside the MPI attractor from real-time forecasts at present.

It is seen in Fig. 5 that while the five models behave differently at first, all models quickly converge to a similar intensity error of $\sim 8 \mathrm{~m} \mathrm{~s}^{-1}$ after 3 days. The strong intensity variations obtained from the RE model and the HSD model offer a hint for this real-time intensity error growth. Specifically, for the global models whose initial hurricane intensity is much weaker than the observed intensity, the hurricane initial states are essentially outside the MPI attractor (i.e., the initial vortex intensity is too weak). As a result, they will be immediately pulled into the MPI attractor, thus accounting for the decrease of the intensity errors during the first 3 days into integration, similar to the behaviors drawn from the HSD model (Fig. 1) and the RE model (Fig. 3). Because of the

\footnotetext{
${ }^{5}$ Given the absolute intensity errors in the range of $8-10 \mathrm{~m} \mathrm{~s}^{-1}$ the intensity threshold that represents more accurately the lower bound of the MPI attractor would be in the range of $55-57 \mathrm{~m} \mathrm{~s}^{-1}$ (category 3 and above). Because of the limited sample size, the threshold of $47 \mathrm{~m} \mathrm{~s}^{-1}$ is chosen for the strong storm verification in this study. Verifications for a higher threshold of $55 \mathrm{~m} \mathrm{~s}^{-1}$ give similar results as shown in Fig. 5, except for a smaller number of verified cases and so are of less statistical significance.
}

finite volume of the MPI attractor, the hurricane states are subsequently trapped in the MPI attractor, and their intensity errors are thus bounded. Given that the size of the MPI attractor as projected onto the $V_{m}$ dimension is $\sim 8 \mathrm{~m} \mathrm{~s}^{-1}$ as obtained from the RE model, one can see that the $8 \mathrm{~m} \mathrm{~s}^{-1}$ limit appears to be indeed realized for these global model forecasts.

For the regional models that employ a vortex initialization procedure to match the initial intensity with the observed intensity, the intensity errors also spread out rapidly over the entire MPI attractor after $\sim 25 \mathrm{~h}$ into integration. Although the error growth in the regional models is less than that in the global models, the rapid intensity error growth during the transient period in all regional models is an indication that the consistency among $\left(V_{m}, W_{m}, B_{m}\right)$ may not be ensured during the vortex initialization process, which leads to strong wandering of the model state, similar to what is shown in Figs. 1 and 3. Note that unlike the error growth in the Hurricane Weather Research and Forecasting (HWRF) Model, whose intensity does not experience a surge in error growth during the first $12 \mathrm{~h}$ probably due to its balanced bogus vortex technique (Tallapragada et al. 2014b), the more rapid error growth in the other two regional models is likely because these models seem to constrain only the maximum surface wind to match the observed $V_{m}$ rather than the full vortex structure. As such, the initial hurricane states in the Geophysical Fluid Dynamics Laboratory (GFDL) model and the tropical cyclone version of the Coupled Ocean-Atmosphere Mesoscale Prediction System (COAMPS-TC) model may be actually far from the MPI attractor (in the dimensions of $W_{m}$ and $B_{m}$ ), thus giving rise to the rapid error growth before spiraling toward the MPI attractor similar to the experiments with the HSD model and the RE model. Of course, the above-mentioned explanations of the error growth in both the regional and global models are more or less speculative until more detailed analyses of the dynamics for each individual model are examined. However, the general behaviors of the rapid error growth and the subsequent saturation in these operational models at the 
same limit of $8 \mathrm{~m} \mathrm{~s}^{-1}$ after approximately 3 days are quite consistent with both the RE and HSD models, thus offering new insight into the error growth issue in operational models.

From the perspective of real-time error growth shown in Figs. 2 and 5, these verifications demonstrate an important aspect of the intrinsic predictability limit of the hurricane's intensity. If one takes the limit of $8 \mathrm{~m} \mathrm{~s}^{-1}$ obtained from the RE model at face value as a real-time error saturation threshold, it is immediately suggestive from Figs. 2 and 5 that it would take less than 3 days for hurricanes to approach this error saturation limit in real-time intensity forecasts. In fact, Fig. 5 shows further that it takes even less time $(20-27 \mathrm{~h})$ for hurricanes to reach this error saturation level once they are of category 2 and above, which is again consistent with the chaotic property of the MPI attractor demonstrated in the RE model. Therefore, we speculate that the 3-day interval is likely the maximum range that a dynamical model could be most skillful in predicting hurricane intensity. After the 3-day limit, the intensity errors will stay at $\sim 8 \mathrm{~m} \mathrm{~s}^{-1}$ at longer ranges, provided that the environmental conditions are sufficiently favorable for the error saturation limit to reach this saturation threshold. Of course, this postulation is by no mean conclusive, as it is based on the error saturation of the maximum tangential wind error that may not reflect the entire TC dynamics. One could have a longer predictability range for forecasts of, say, the intensity tendency or the rapid intensification. However, addressing such different aspects of intensity forecasts would require much more in-depth investigation of TC dynamics than what is presented in this study.

Despite the consistent behaviors of the real-time intensity error growth with those inferred from the HSD and RE models, we should point out that the above-mentioned real-time error growth analysis contains some inherent uncertainties due to several factors, such as a limited sample size, a lack of detailed analyses of the model vortex's initial condition, inadequate representation of hurricane physics, and even discrepancies between the model pointwise maximum surface wind and the observed intensity. Furthermore, our real-time error analysis in Fig. 5 is only for the initially strong storms of category 2 and above. A caveat of this real-time statistics for initially strong storms is that once hurricanes reach category 2 and above, rarely do they sustain high intensity for five full days, because in most cases they weaken quickly due to making landfall or curving to a more hostile environment at higher latitudes. Therefore, the intensity statistics for the initially strong storms at lead times longer than 3 days is heavily influenced by landfalling and other unfavorable conditions, and one should be therefore cautioned when judging the statistics of the intensity errors beyond the 3-day lead time for strong hurricanes.

As a final note, we should mention that, unlike the behaviors of strong storms, whose states are close to the MPI attractor, an initially weak storm may have distinct error growth characteristics, which are related to the transient orbit and so differ fundamentally from the error growth inside the MPI attractor (Lorenz 1963). This explains the longer time interval needed to reach the error saturation shown in Fig. 2 ( $\sim 3$ days) than what is shown in Fig. 5 ( 1 day). Such error growth, which is related to transient orbits in real-time forecasts, requires more analyses of flow orbits than a few simple statistics, and it is not examined herein due to our lack of linear tangential models.

DISCUSSION. While the existence of a chaotic MPI attractor imposes a limit of $\sim 8-10 \mathrm{~m} \mathrm{~s}^{-1}$ on the hurricane intensity errors at the range of $4-5$ days, there is still a question of whether this is a true value for the hurricane intensity error saturation. Previous studies have shown that such intensity error saturation may depend on specific environmental conditions, the type of numerical models, or physical approximations. For example, a 500-day idealized experiment in the study by Hakim (2011) using a different axisymmetric model captured intensity variation as large as $12 \mathrm{~m} \mathrm{~s}^{-1}$ at the statistical equilibrium, whereas numerical simulations with a full-physics model by Yang et al. (2007) exhibited a smaller variation of $\sim 7 \mathrm{~m} \mathrm{~s}^{-1}$ at the mature stage (cf. Fig. 1 in Yang et al. 2007). Likewise, real-time statistics of the HWRF Model shows slightly smaller 4-5-day errors in the eastern Pacific basin (7-9 $\mathrm{m} \mathrm{s}^{-1}$ ) and in the North Atlantic basin (8-10 $\mathrm{m} \mathrm{s}^{-1}$; Tallapragada et al. 2014a). Despite these ranges of intensity error saturation limit in different basins and modeling systems, it is important to note that the convergence of the absolute intensity errors in the range of 8-10 $\mathrm{m} \mathrm{s}^{-1}$ from different models and real-time statistics suggests that we are likely close to a true intensity error saturation limit below which the intensity error may not be reduced further after 4-5-day lead times.

Given the intensity error saturation of $\sim 8 \mathrm{~m} \mathrm{~s}^{-1}$ as drawn from both the RE model and real-time experiments, it is important to note that this error saturation alone is not sufficient to determine the range of predictability. In principle, it is possible that hurricane development may take a long time to reach a saturation threshold and so the existence of the error 
saturation does not contain information of how long the saturation level will be reached. Furthermore, the $8 \mathrm{~m} \mathrm{~s}^{-1}$ limit is obtained under an assumption that favorable environmental conditions for hurricane development are well maintained such that the MPI attractor could be realized. In this regard, the limit of $8 \mathrm{~m} \mathrm{~s}^{-1}$ simply establishes an intrinsic intensity difference that two arbitrarily different initial vortex states would have at the maximum intensity stage.

It should be also mentioned that the range of hurricane intensity predictability, which is defined here as a time interval for an initial intensity error to approach the error saturation of $8 \mathrm{~m} \mathrm{~s}^{-1}$, may vary from about 3 days at the early developing stage to less than 1 day at the mature stage. Such an estimation of the range of predictability of about 3 days is not derived or proven by any model; it is merely obtained from the statistics of real-time intensity forecasts, similar to the 2-week limit in Lorenz's model that is obtained from the assumption of an empirical $-5 / 3$ spectrum. This 3-day range of predictability is consistent with recent error variance analysis from a linear inverse model by Hakim (2013), which also suggested a limit of 3 days for forecasts of tangential wind at the quasistationary equilibrium stage. Based on these results, it is rational to postulate that

1) the $8 \mathrm{~m} \mathrm{~s}^{-1}$ error is close to a lower bound of the absolute intensity errors at $4-5$-day lead times that we may not be able to reduce further in the future; and

2) the maximum range of predictability of hurricane intensity is about 3 days.

We emphasize again that the above postulation of a 3-day predictability limit is solely from the perspective of intensity absolute errors. It is possible that forecasts of the phase of hurricane development such that the rapid intensification or the rapid weakening can be more predictable. Likewise, a specific numerical model may have a longer or shorter range of predictability, depending on each model system design and configuration, that we need to examine individually.

Although our result suggests that improving the hurricane intensity at the 4-5-day lead times ought to focus more on the large-scale environment, we by no means dismiss the importance of the vortex's initial condition. This is because an initially too-weak or too-strong storm could certainly determine the subsequent feedback in some specific situations. For example, a too-weak storm could be sheared off quickly during initial development, such that the WISHE feedback might not have enough time to become effective, or that dry-air intrusion could severely interfere with the storm's development with the result that the necessary equilibrium mechanisms could never be applied. The conclusion in this study about the longer-range control of the environment to the hurricane development is under an implicit assumption of well-maintained favorable conditions for hurricanes to develop, and it should be interpreted therefore from the statistical perspective instead of from the development of a single storm.

ACKNOWLEDGMENTS. We thank Greg Hakim (University of Washington), two anonymous reviewers, and Editor Chris Landsea for their constructive comments and suggestions, which helped improve the presentation of this manuscript substantially. This research was supported by the start-up fund provided by Indiana University in Bloomington, Indiana, and partially by the NOAA Hurricane Forecast Improvement Program (HFIP).

\section{REFERENCES}

Brown, B. R., and G. J. Hakim, 2013: Variability and predictability of a three-dimensional hurricane in statistical equilibrium. J. Atmos. Sci., 70, 1806-1820, doi:10.1175/JAS-D-12-0112.1.

Bryan, G. H., and R. Rotunno, 2009: The maximum intensity of tropical cyclones in axisymmetric numerical model simulations. Mon. Wea. Rev., 137, 1770-1789, doi:10.1175/2008MWR2709.1.

DeMaria, M., and W. H. Schubert, 1985: Axisymmetric, primitive equation, spectral tropical cyclone model. Part II: Normal mode initialization. J. Atmos. Sci., 42, 12251236, doi:10.1175/1520-0469(1985)042<1225:APEST C>2.0.CO;2.

Durran, D. R., and M. A. Gingrich, 2014: Atmospheric predictability: Why butterflies are not of practical importance. J. Atmos. Sci., 71, 2476-2488, doi:10.1175/JAS-D-14-0007.1.

Emanuel, K. A., 1986: An air-sea interaction theory for tropical cyclones. Part I: Steady-state maintenance. J. Atmos. Sci., 43, 585-605, doi:10.1175/1520-0469(1986)043<0585:AASITF $>2$ .0.CO;2.

- , 2000: A statistical analysis of tropical cyclone intensity. Mon. Wea. Rev., 128, 1139-1152, doi:10.1175/1520-0493(2000)128<1139:ASAOTC $>2.0 . \mathrm{CO} ; 2$.

—, 2003: Tropical cyclones. Annu. Rev. Earth Planet. Sci., 31, 75-104, doi:10.1146/annurev.earth .31.100901.141259. 
Hakim, G. J., 2011: The mean state of axisymmetric hurricanes in statistical equilibrium. J. Atmos. Sci., 68, 1364-1376, doi:10.1175/2010JAS3644.1.

—, 2013: The variability and predictability of axisymmetric hurricanes in statistical equilibrium. J. Atmos. Sci., 70, 993-1005, doi:10.1175/JAS-D-12-0188.1.

Henderson-Sellers, A., and Coauthors, 1998: Tropical cyclones and global climate change: A post-IPCC assessment. Bull. Amer. Meteor. Soc., 79, 19-38, doi:10.1175/1520-0477(1998)079<0019:TCAGCC $>2.0 . \mathrm{CO} ; 2$.

Hendricks, E. A., M. T. Montgomery, and C. A. Davis, 2004: The role of "vortical" hot towers in the formation of Tropical Cyclone Diana (1984). J. Atmos. Sci., 61, 12091232, doi:10.1175/1520-0469(2004)061<1209:TROV $\mathrm{HT}>2.0 . \mathrm{CO} ; 2$.

Judt, F., S. S. Chen, and J. Berner, 2016: Predictability of tropical cyclone intensity: Scale-dependent forecast error growth in high-resolution stochastic kineticenergy backscatter ensembles. Quart. J. Roy. Meteor. Soc., 142, 43-57, doi:10.1002/qj.2626.

Kieu, C. Q., 2015: Hurricane maximum potential intensity equilibrium. Quart. J. Roy. Meteor. Soc., 141, 2471-2480, doi:10.1002/qj.2556.

Knutson, T. R., and Coauthors, 2010: Tropical cyclones and climate change. Nat. Geosci., 3, 157-163, doi:10.1038/ngeo779.

Lorenz, E. N., 1963: Deterministic nonperiodic flow. J. Atmos. Sci., 20, 130-141, doi:10.1175 /1520-0469(1963)020<0130:DNF>2.0.CO;2.

— 1969: The predictability of a flow which possesses many scales of motion. Tellus, 21A, 289-307, doi:10.1111/j.2153-3490.1969.tb00444.x.

Ott, E., 2002: Chaos in Dynamical Systems. 2nd ed. Cambridge University Press, 492 pp.

Palmer, T. N., 1993: Extended-range atmospheric prediction and the Lorenz model. Bull. Amer. Meteor. Soc., 74, 49-65, doi:10.1175/1520-0477(1993)074<0049:ER APAT $>2.0 . \mathrm{CO} ; 2$.
Rotunno, R., and K. A. Emanuel, 1987: An air-sea interaction theory for tropical cyclones. Part II: Evolutionary study using a nonhydrostatic axisymmetric numerical model. J. Atmos. Sci., 44, 542-561, doi:10.1175/1520-0469(1987)044<0542:AAITFT >2 .0.CO;2.

— , and C. Snyder, 2008: A generalization of Lorenz's model for the predictability of flows with many scales of motion. J. Atmos. Sci., 65, 1063-1076, doi:10.1175/2007JAS2449.1.

Tallapragada, V., C. Q. Kieu, Y. Kwon, S. Trahan, Q. Liu, Z. Zhang, and I.-H. Kwon, 2014a: Evaluation of storm structure from the operational HWRF during 2012 implementation. Mon. Wea. Rev., 142, 4308-4325, doi:10.1175/MWR-D-13-00010.1.

— Research and Forecasting (HWRF) model: 2014 scientific documentation. NCAR Development Testbed Center Rep., 105 pp. [Available online at www.dtcenter .org/HurrWRF/users/docs/scientific_documents /HWRFv3.6a_ScientificDoc.pdf.]

Walsh, K., and Coauthors, 2015: Hurricanes and climate: The U.S. CLIVAR Working Group on Hurricanes. Bull. Amer. Meteor. Soc., 96, 997-1017, doi:10.1175 /BAMS-D-13-00242.1.

Wang, Y., 2001: An explicit simulation of tropical cyclones with a triply nested movable mesh primitive equation model: TCM3. Part I: Model description and control experiment. Mon. Wea. Rev., 129, 1370-1394, doi:10.1175/1520-0493(2001)129<1370:AESOTC >2 .0.CO;2.

Willoughby, H. E., 1979: Forced secondary circulations in hurricanes. J. Geophys. Res., 84, 3173-3183, doi:10.1029/JC084iC06p03173.

Yang, B., Y. Wang, and B. Wang, 2007: The effect of internally generated inner-core asymmetries on tropical cyclone potential intensity. J. Atmos. Sci., 64, 1165-1188, doi:10.1175/JAS3971.1. 
AMS titles now

available as eBooks

at springer.com

\section{AMS BOOKS}

\section{RESEARCH APPLICATIONS HISTORY}

www.ametsoc.org/amsbookstore

Springer

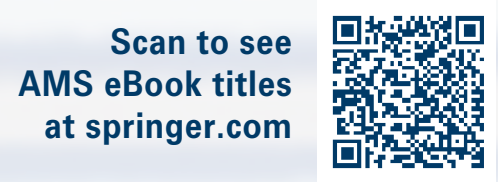

Springer

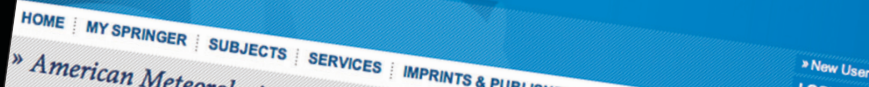

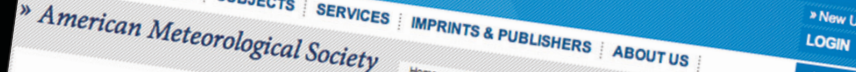

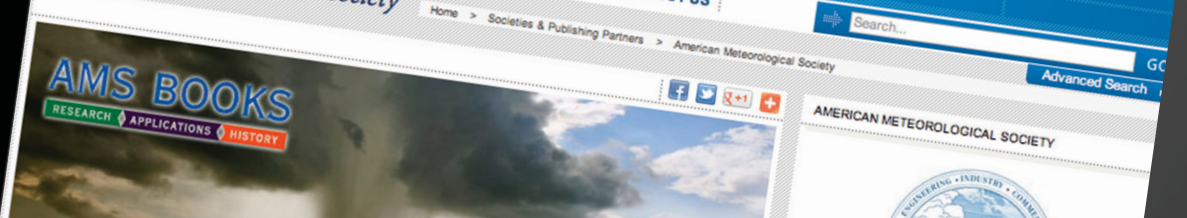

$x^{2}+5=0$

American Meteorological Society

Promoting the development

the atmospheric and rethent and dise

Whatic and related sciences.

American Meteorological Society eBot .

AMs

published by the AMS a monograph publishing pred now

shing program related o ho poer-

Dses a serios

related to th as eBooks) as weil ar ariy out-of-print vol metoorological and historica journals

to the atmospheric sciences a growing list of acades of which have been uroughonographs

ing

Sort listing by:

AMERICAN OR: ORolevance

वध्रि Export to $\mathrm{cs}$

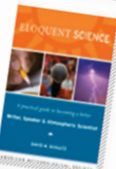

Eloquent Science

Hoquent Scies

A Practical Guit

2009

Price from $\$ 45.00$

iecome an AMS Member

Avaliablie Format.

A MER ICA N METEOROLOG ICAL SOCIETY 